\title{
High-Resolution Chemical-state Mapping and Analysis for Nuclear Safeguards with Microcalorimeter SEM-EDS
}

\author{
Matthew Carpenter ${ }^{1}$, Mark Croce ${ }^{2}$, Chandler Smith ${ }^{2}$ and Katrina Koehler ${ }^{2}$ \\ ${ }^{1}$ Safeguards Science and Technology Nuclear Engineering and Nonproliferation, Los Alamos National \\ Laboratory, Los Alamos, New Mexico, United States, ${ }^{2}$ Los Alamos National Laboratory, Los Alamos, \\ New Mexico, United States
}

Low-temperature microcalorimeter x-ray detectors based on transition-edge sensors (TESs) integrated with scanning electron microscopes (SEMs) can combine the resolution of wavelength-dispersive spectroscopy (WDS) instruments with a broad energy range and efficiency comparable to energydispersive spectroscopy (EDS) instruments [1]. With recent developments in microwave multiplexing of hundreds of TES pixels [2] and the availability of lower-cost, reliable dilution refrigerator cryostats, it is now becoming practical to measure chemical speciation in the SEM via subtle peak shifts in the emission spectrum [3]. Microcalorimeters can also improve sensitivity for elemental composition. Due to the Bremsstrahlung background and $\sim 100 \mathrm{eV}$ energy resolution of the Si drift detector (SDD), standard EDS has detection limits of order $0.1 \mathrm{wt} \%$, while WDS has of order $0.01 \mathrm{wt} \%$ detection limit owing to its higher resolution ( 5-15 eV FWHM) [4,5]. With even higher energy resolution than typical WDS instruments, microcalorimeters have the potential to further improve detection limits especially when spectral interferences are present.

We are designing a TES detector integrated with a JEOL JSM-7200F SEM to take advantage of these improvements. The goal is to develop a nanoscale chemical speciation mapping capability with a focus on materials for nuclear safeguards. The target resolution is better than $3 \mathrm{eV}$ full-width at half-maximum (FWHM) at $3 \mathrm{keV}$ near the uranium $\mathrm{M}$ lines, allowing both greater sensitivity to trace amounts of analyte as well as providing chemical information such as oxidation state from minor spectral details (Fig. 1, left). The detector will have 256 TES pixels on the end of a snout for insertion into the sample chamber for geometric efficiency similar to typical EDS detectors. The right panel of Fig. 1 shows a spectrum of $\mathrm{Cs}_{2} \mathrm{UO}_{2} \mathrm{Cl}_{4}$ simulated with the Monte-Carlo SEM simulation software DTSA-II [6] accounting for the expected parameters of the future TES detector including windows/filters and pixel size and composition. Minor $\mathrm{U} \mathrm{M}$ lines are clearly resolved from $\mathrm{Cl} \mathrm{K}$ lines in 30 seconds of acquisition time as shown in the figure inset, demonstrating the efficiency and sensitivity of this class of detector.

The primary focus is on the transuranic $\mathrm{M}$ lines between 2 and $5 \mathrm{keV}$ as these may be excited by a lower electron beam energy (down to $5 \mathrm{kV} \mathrm{V}$ acc) yielding a smaller material volume probed by $\mathrm{x}$-ray emission than at higher beam energies. An approximation relating the typical interaction volume to the beam energy and material is given by Goldstein [7]:

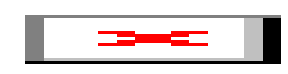

where $\mathrm{E}_{0}$ is the incident electron energy in $\mathrm{keV}, \mathrm{E}_{\mathrm{c}}$ is the energy of the emission line of interest in $\mathrm{keV}, \rho$ is the material density in $\mathrm{g} / \mathrm{cm}^{3}$, and the range $\mathrm{R}$ is in $\mu \mathrm{m}$. From this relation we estimate the range in $\mathrm{UO}_{2}$ to be $\sim 50 \mathrm{~nm}$ for the $\mathrm{U} \mathrm{M}_{\alpha}$ line excited by $5 \mathrm{keV}$ electrons and $\sim 25 \mathrm{~nm}$ for the $\mathrm{U} \mathrm{L}_{\alpha}$ line excited by 20 $\mathrm{keV}$ electrons. For sub-micron size scales the lower energy actinide $\mathrm{M}$ lines therefore provide better spatial resolution than their $\mathrm{L}$ lines. The sub-10 eV resolution of TES detectors is able to cleanly resolve 
transuranic $M$ lines from one another in a mixed sample, opening this size scale up to an array of safeguards applications at the nanometer scale.

A challenge to the implementation of TES detectors for high-resolution EDS analysis is in accurate energy calibration and tracking of time-dependent gain drift. TESs are highly nonlinear detectors compared to typical solid-state detectors such as silicon drift detectors, and the energy calibration may change over timescales of minutes due to multiple factors. This may be tracked by frequently referencing a calibration standard with a large number of emission lines spanning the energy range, interleaving the calibration data with the sample data to be later deconvolved in the pulse processor to extract the sample data with corrections from the calibration reference. The system under development at LANL will integrate the calibration routines with detector and SEM control to account for these factors and produce highresolution $\mathrm{X}$-ray emission spectra in real time to allow chemical state mapping of nanoscale samples.
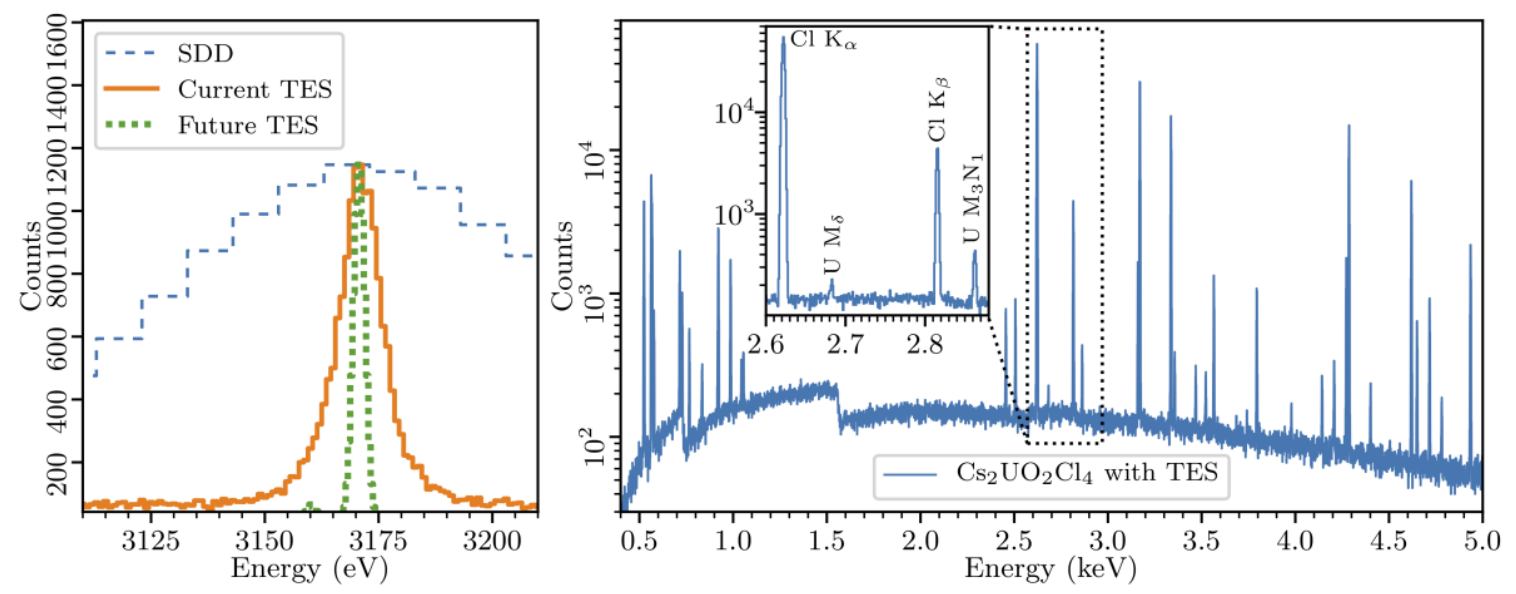

Figure 1. Fig. 1: Left: Comparison of energy resolution at uranium $\mathrm{M} \alpha$ line measured with an SDD and an existing TES detector on a SEM compared to simulated spectrum of TES detector under development at LANL. Right: Simulated x-ray spectrum of Cs2UO2Cl4 with future TES detector of 3 eV FWHM resolution. This spectrum represents a 30 second acquisition with a 256 pixel TES detector at $40 \mathrm{~mm}$ standoff and a beam current of $20 \mathrm{nA}$ at $10 \mathrm{kV}$ Vacc.

\section{References}

[1] D.A. Wollman, K.D. Irwin, G.C. Hilton, L.L. Dulcie, D.E. Newbury, J.M. Martinis, J. Microscopy 188(3), 196-223 (1997), DOI: 10.1046/j.1365-2818.1997.2670824.X

[2] J.D. Gard, D.T. Becker, D.A. Bennett, J.W. Fowler, G.C. Hilton, J.A.B. Mates, C.D. Reintsema, D.R. Schmidt, D.S. Swetz, J.N. Ullom, J. Low Temp. Phys. 193, 485-497 (2018), DOI: 10.1007/s10909-018-2012-2 [3] R. Cantor, M.P. Croce, G.J. Havrilla, M.H. Carpenter, K. McIntosh, J.A. Hall, S.A. Kozimor, H. Naito, M.W.M.W. Rabin, D.R. Schmidt, D. Swetz, J.N. Ullom, Microsc. Microanal. 22, 434-435 (2016), DOI:10.1017/S1431927616003020

[4] D.E. Newbury, N.W.M. Ritchie, J. Mater. Sci. 50, 493-518 (2014), DOI:10.1007/s10853-014-8685-2

[5] P. Kuisma-Kursula, X-Ray Spectrom. 29, 111-118 (2000), DOI:10.1002/(SICI)10974539(200001/02)29:1<111::AID-XRS408 >3.0.CO;2-W

[6] N.W.M. Ritchie, NIST DTSA-II software, http://www.cstl.nist.gov/div837/837.02/epq/dtsa2/index.html [7] J.I. Goldstein, D.E. Newbury, D.C. Joy, C.E. Lyman, P. Echlin, E. Lifshin, L. Sawyer, J. Michael, Scanning electron microscopy and x-ray microanalysis, Third Edition, 2003. DOI:10.1007/978-1-4615-0215-9 